\title{
Controlling 3D Percolation Threshold of Conductor-Insulator Composites by Changing the Granular Size of Conductors
}

\author{
Kazuhito Shida, Ryoji Sahara, Hiroshi Mizuseki and Yoshiyuki Kawazoe \\ Institute for Materials Research, Tohoku University, Sendai 980-8577, Japan
}

The percolation threshold on a cubic lattice is estimated under a binary distribution of the sizes of the conducting particles. Although we have already investigated a similar size distribution model in $2 \mathrm{D}$ (where no drastic change in their percolation threshold was observed) (K. Shida, R. Sahara, M. N. Tripathi, H. Mizuseki and Y. Kawazoe: Mater. Trans. 50 (2009) 2848-2851), a 3D version of this model has not been reported before. In the $3 \mathrm{D}$ case, a significant decrease in the percolation threshold compared to the monodisperse cases is observed. [doi:10.2320/matertrans.M2011176]

(Received June 20, 2011; Accepted September 26, 2011; Published November 16, 2011)

Keywords: thin film conductor, percolation model, critical phenomenon, granular material

\section{Introduction}

The percolation model ${ }^{1)}$ is a simple and powerful theoretical tool that plays an important role in explaining various physical phenomena related to the properties of disordered systems. Recently, a number of interesting modifications have been added to this simple model, drawing increased attention. In our previous works, ${ }^{2,3)}$ we proposed a new type of 2D site-percolation model, where two different sizes of particles, labeled A and B, are randomly placed on a lattice, and we investigated the model by the Monte Carlo approach. Despite a clear difference from in the particle size distribution, the percolation threshold $p_{\mathrm{c}}$ did not exhibit a large deviation from that of the conventional 2D percolation model without particle size distribution.4) Other authors reported results basically in accordance to ours. ${ }^{5,6)} \mathrm{We}$ then focused on a similar problem, but in terms of the magnitude of electronic conductance, on the 3D lattice, that can be evaluated by a random resistor network model and the Kirchoff equation. The results ${ }^{4)}$ suggested that in the 3D case, binary distributions in the sizes of the particles may change the percolation threshold. These results demonstrate that the introduction of particle size distribution into a simple percolation system exerts a complex and non-trivial effect ${ }^{7)}$ that is difficult to be predicted by simple empirical models ${ }^{8,9)}$ depending on the dimensionality of the space.

This peculiar effect has never been investigated in the most basic 3D setting of a percolation model, such as the cubic lattice. Although many interesting results have been reported with 2D off-lattice systems, many of the studies did not properly treat the excluded-volume effect among particles (e.g., permeable ellipse models ${ }^{10,11)}$ ). Along with a more realistic selection of dimensionality $(D=3)$, we believe the excluded-volume effect among particles on modulated percolation problems has too large of an impact to deny. Therefore, in this paper we carried out a large-scale computational experiment of 3D impermeable particles to confirm the size modulation's effect on the basic percolation phenomena in terms of connectivity. The type of size modulation tested is also the most basic one: a binary size distribution of "conducting" particles.
Why is such an estimation of $p_{\mathrm{c}}$ important? The percolation behavior of composite materials has been explained accurately by a simple theoretical model, such as the connectivity between randomly occupied sites (or bonds) on a lattice. This success, in turn, means that the limits of composite materials imposed by the percolation transition are rather mathematical and difficult to overcome by the conventional methodologies of material sciences. In particular, the concept of a universality class ${ }^{1,12}$ suggests that the percolation limit is strongly restricted by the dimensionality of the materials. Therefore, the composite materials in bulk (3D) and film (2D) materials would have their respective limits. On the other hand, although there are a number of physically realizable methods ${ }^{13-15)}$ to obtain similar effects, the most straightforward way to modify the behavior of percolating systems is to introduce particle size distributions by simply mixing two different types of particles. Therefore it is important to know how percolation is affected by different particle size distributions in 3D cases that assume finite and thus more realistic material thickness, compared to $2 \mathrm{D}$ cases.

\section{Methods}

We studied the site-percolation model on $L \times L \times L$ cubic lattices, with some conducting particles (total volume fraction of the conducting particles is $p$ ) being larger than others, using the following method. Three different sizes of particles, $1 \times 1 \times 1,2 \times 2 \times 2$ and $3 \times 3 \times 3$, were considered. The size of a particle is referred to as $X$, therefore $X=1,2$ and 3: we deal with the mixtures of $X=1,2$ or $X=1,3$ (see Fig. 1). A series of algorithms, basically the same as the ones we used for the 2D problem, is used to generate random configuration ensembles of these cubes with size variations. First, according to the values of $p, X, L$ and the fraction ratio $\theta$ (introduced below), the numbers of particles with unit and increased sizes are calculated. Then, non-overlapping $X^{3}$ cubes with the required number and size are randomly placed on the lattice by means of a simple trial-and-error algorithm to decide the positions of large particles. On the lattice sites not occupied by these large particles, the 

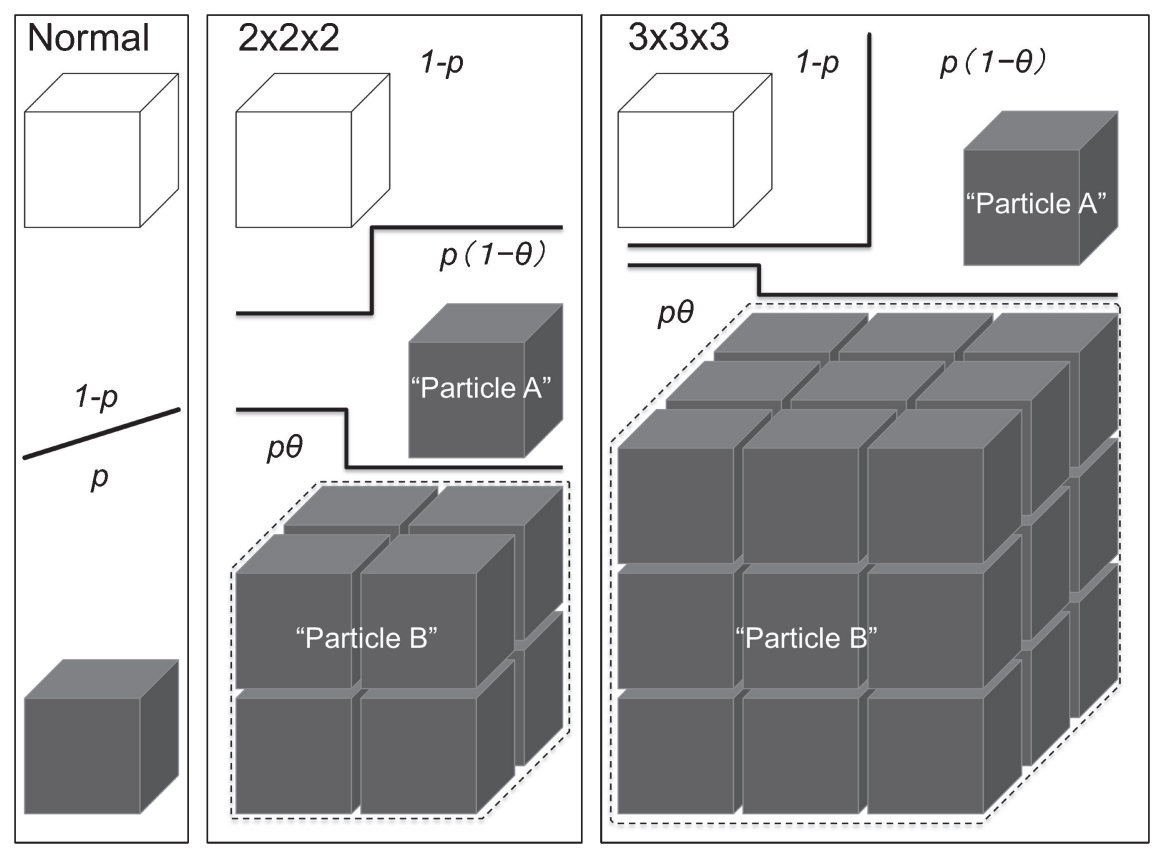

Fig. 1 In this work, these particle designs are used to simulate the size variations of the conducting materials. In this figure, the white sites are insulating sites whereas the black sites are conducting sites. Among all conducting sites, varying fractions $(\theta=0.3,0.5$ and 0.7$)$ are arranged in $2 \times 2 \times 2$ and $3 \times 3 \times 3$ configurations and are regarded as particles of larger sizes (particle B).

conductor or insulator particles with unit size are randomly placed. This simple scheme apparently samples all possible configurations with given combinations of the number and type of particles. Finally, a standard procedure for cluster analysis is performed to obtain the connected cluster size statistics. This procedure is repeated for 50000 different random particle configurations per condition. For each condition introduced below, lattice sizes $L=32 \sim 256$ are simulated. The simulated range of $p$ is set in such a way that the values of $p_{c}$ (estimated roughly by a number of preliminary simulations) are confined within the range. Then a number of simulations are carried out to sweep the range of $p$ with a 0.02 interval. Shorter intervals were desirable for the precise determination of $p_{\mathrm{c}}$, even if the precision can be augmented by the finite scaling analysis. However, due to the size of the computational resource required (one $L=256$ case needed as much as $258 \mathrm{~h}$ to finish), 0.02 was the shortest interval we could use. However, this precision is sufficient to discuss the relationship between the magnitude of $p_{\mathrm{c}}$ reduction effect and the mixture ratio of the bigger particles, which is one focus of the current report.

The volume fraction of the bigger particles over all conducting particles, denoted as $\theta$, is also a controlled parameter: we have tested three different series of simulations in which $\theta=0.3,0.5$ and 0.7 is used. For example, when $X=1$ and $X=2$ particles are mixed with the $\theta=0.3$ condition, their volume fraction ratio and number ratio are $7: 3$ and $70: \frac{30}{8}$, respectively. The conventional site-percolation model is of course the $\theta=0$ case.

The $\theta>0.7$ situation is quite interesting but very difficult to reach, in particular when $p$ is also large. In the limit of $\theta \rightarrow 1.0$ and $p \rightarrow 1.0$, the system consists almost entirely of bigger particles. There are many ways to "pack" those bigger particles in a lattice other than a simple cubic configuration, including configurations analogous to hexagonal closepacking (hcp). However, it should be remembered that hcp of the sphere can be affected in a very complex manner by a small number of smaller particles. ${ }^{16,17)}$ In the case of closepacked cubes toward $\theta \rightarrow 1.0$ and $p \rightarrow 1.0$ too, a few small (unit size) cubes scattered over the lattice may make the packing problem dramatically difficult. Due to these difficulties in efficiently sampling various packing configurations near $\theta=1.0$, we have to choose $\theta=0.7$ as our limit of bigger particle volume fraction.

The $\theta=1.0$ case is merely one of many possible combinations of particle size distributions under which percolation phenomena can be affected in a special manner. For example, in our previous work, size modulation in the insulator particles dramatically affected 2D percolations. ${ }^{18)}$ The effect of insulator size modulation in $3 \mathrm{D}$ cases is of course interesting, but we confine ourselves to the simplest size modulations introduced in an independent manner. This is mainly because too many combinations of particle size distributions are possible in the system, when insulators and conductor particles may have independent size distributions. To investigate this vast number of potential combinations in a more organized manner, we have been working on a systematic method (manuscript in preparation) to explore the various types of modulations of percolation problems.

The output of these calculations is the mean finite cluster size, $S(L, p)$, defined as a summation taken for all clusters except the largest one,

$$
S(L, p)=\sum_{s} s^{2} n_{s}(p),
$$

where $n_{s}(p)$ is the average number of clusters with size $s$. In short, the peak of the finite cluster size gives us an estimate of the $p_{\mathrm{c}}$ of the percolation, although a better precicion is possible by means of the finite scaling analysis. 

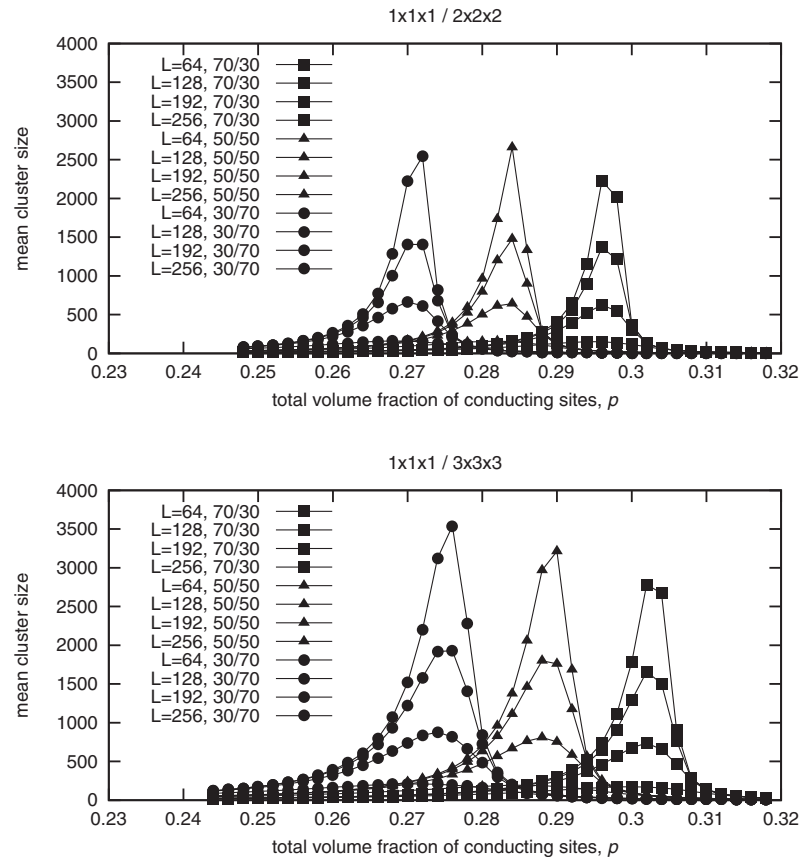

Fig. 2 The mean size of finite connected clusters as a function of the volume fraction of conducting sites on a 3D lattice, plotted for $L=160$, 192, 224 and 256, is shown for $X=1,2$ mixture (above) and $X=1,3$ mixture (lower). The leftward shift of the peaks of the plots clearly indicates the $p_{\mathrm{c}}$ reduction effect of bigger conductor particles.

\section{Results}

The location of the percolation threshold, $p_{\mathrm{c}}$, is strongly affected by existence of the bigger particles, as clearly demonstrated by plots of the whole density of conductor particles (X-axis) versus the mean size of finite connected clusters (Y-axis) in Fig. 2. In this figure, the location of the percolation threshold, $p_{\mathrm{c}}$ is indicated by the peak of the plot: each condition shows four peaks with different heights and grades of steepness, corresponding to four different lattice sizes ( $L=256$ shows the highest and steepest plots). Note that, just like $2 \mathrm{D}$ percolation systems with correlations in site placement, the general behavior of the percolation does not seem to be changed by the introduction of bigger particles (otherwise, there should be distinguishable changes in the shape of the plot depending on $\theta$ ). The introduction of bigger conductor particles do have a strong effect of $p_{\mathrm{c}}$ reduction. However, contrary to our intuitive expectation, the effect of the reduction decreases when the size of bigger particles are increased; $3 \times 3 \times 3$ particles have weaker effect of $p_{\mathrm{c}}$ reduction than $2 \times 2 \times 2$ particles. Compared to our $2 \mathrm{D}$ result ${ }^{3)}$ reported before, these results exhibit a stark contrast: in $2 \mathrm{D}$ site percolation problems in square lattices, the binary size distributions of particles resulted in a barely observable change in $p_{\mathrm{c}}$.

Then how does the magnitude of reduction depend on the amount of bigger particles? In Fig. 3, the location of the percolation threshold is indicated as a function of the fraction of bigger particles. As intuitively expected, the greater the fraction of bigger particles in the system, the lower $p_{\mathrm{c}}$ becomes. The quantitative dependence is much simpler than

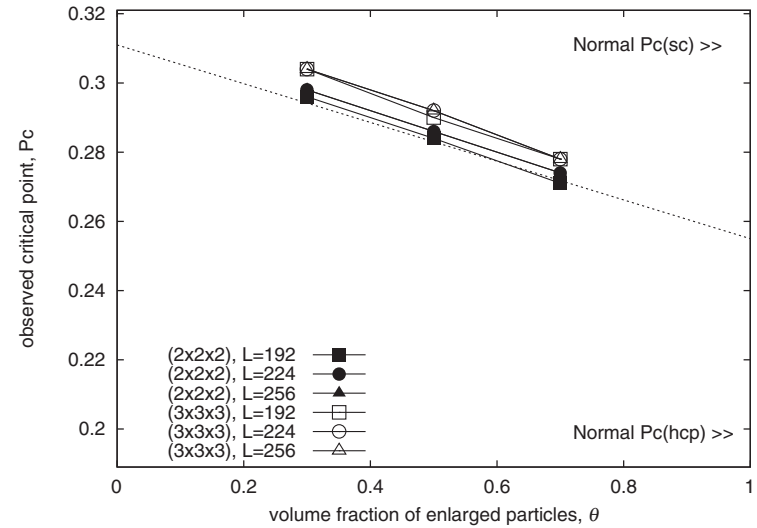

Fig. 3 The estimated values of $p_{\mathrm{c}}$ for $L=64,128,192$ and 256 are shown as functions of the fraction of an bigger particle, $\theta$. Note the linear dependence of the shift of $p_{\mathrm{c}}$ on the $\theta$ and the limiting value of $p_{\mathrm{c}}$ toward the left and right limits of $\theta$.

we expected, as the magnitude of this $p_{\mathrm{c}}$ reduction effect seems to be roughly proportional to the fraction of bigger particles. The system seems to exhibit a rather precise convergence to normal percolation ${ }^{19,20)}\left(p_{\mathrm{c}}=0.311\right)$ toward the $\theta \rightarrow 0.0$ limit. This convergence is less accurate when $3 \times 3 \times 3$ particles are used, but this can be addressed by the slightly insufficient system size compared to the size of bigger particles. One thing notable on this result is that the system is apparently not converging to either normal percolation with reduced system size (simple cubic, $p_{\mathrm{c}}=$ $0.311)$ nor site percolation on the hcp-lattice ${ }^{21)}\left(p_{\mathrm{c}}=0.199\right)$, in the $\theta \rightarrow 1.0$ limit. The $\theta \rightarrow 1.0$ limiting value, that is, an intuitively predicted value of the site percolation threshold when all particles are size $2 \times 2 \times 2$, is curiously close to their midpoint $\left(p_{\mathrm{c}}=(0.311+0.199) / 2=0.255\right)$.

\section{Conclusion}

In the conventional percolation model, complete randomness is assumed in the spatial placement of conducting and insulating sites. In other words, the system is constituted of randomly placed particles of exactly the same size. As a result of this randomness in position and uniformity in size, a rather strict mathematical limit appears in the clustering characteristics of the conducting particles. Recently there have been many proposals, from not only purely theoretical but also applicational standpoints, to break this complete randomness to realize and/or investigate the correlated percolation problems. In this work, the correlation is generated by means of a simple binary particle size distribution. Despite the simplicity of the model and the correlation used (which also means that the realization of the correlation in real material is easy), the results of the present study certainly shed some light on an example of correlated percolations. Moreover, the results presented here are further proof of the possibility of improving electronic conduction for a given amount of conductor material just by modulating the particle size distribution of the conductor: without damaging the conductance in the simulated material, the total volume fraction of conductor is reduced by about $15 \%$ $\left(p_{\mathrm{c}}=0.311 \rightarrow 0.270\right)$. 


\section{Acknowledgement}

This work was supported by the Ministry of Economy, Trade and Industry (METI) under "Development for reduction of Indium in a transparent electrode ITO". The authors gratefully acknowledge the Center for Computational Materials Science at the IMR of Tohoku University.

\section{REFERENCES}

1) D. Stauffer and A. Aharony: Introduction to Percolation Theory, (Taylor and Francis, 1992).

2) R. Sahara, H. Mizuseki, K. Ohno and Y. Kawazoe: Mater. Trans. JIM 40 (1999) 1314-1318.

3) R. Sahara, H. Mizuseki, K. Ohno and Y. Kawazoe: J. Phys. Soc. Japan 68 (1999) 3755-3758.

4) K. Shida, R. Sahara, M. N. Tripathi, H. Mizuseki and Y. Kawazoe: Mater. Trans. 50 (2009) 2848-2851.

5) M. K. Phani and D. Dhar: J. Phys. A 17 (1984) L645.

6) D. Dhar: Physica A 242 (1997) 341-346.

7) R. Ogata, T. Odagaki and K. Okazaki: J. Phys. Condens. Matter 17 (2005) 4531-4538.

8) W. J. Kim, M. Taya, K. Yamada and N. Kamiya: J. Appl. Phys. 83
(1998) 2593-2598

9) N. Lebovka, M. Lisunova, Y. P. Mamunya and N. Vygornitskii: J. Phys. D-Appl. Phys. 39 (2006) 2264-2271

10) R. Consiglio, R. N. A. Zouain, D. R. Baker, G. Paul and H. E. Stanley: Phys. A 343 (2004) 343-347.

11) J. A. Quintanilla and R. M. Ziff: Phys. Rev. E 76 (2007) 051115.

12) S.-K. Ma: Modern Theory of Critical Phenomena, (Addison-Wesley, 1976).

13) N. Johner, C. Grimaldi, T. Maeder and P. Ryser: Phys. Rev. E 79 (2009) 020104.

14) I. Krupa, G. Mikova, I. Novak, I. Janigova, Z. Nogellova, F. Lednicky and J. Prokes: Europ. Polymer J. 43 (2007) 2401-2413.

15) H. B. Dai, H. X. Li and F. H. Wang: Surf. Coat. Technol. 201 (2006) 2859-2866.

16) A. R. Kansal, S. Torquato and F. H. Stillinger: J. Chem. Phys. 117 (2002) 8212-8218.

17) G. W. Delaney, S. Hutzler and T. Aste: Phys. Rev. Lett. 101 (2008) 120602.

18) K. Shida, R. Sahara, M. N. Tripathi, H. Mizuseki and Y. Kawazoe: Mater. Trans. 51 (2010) 1141-1144.

19) J. Skvor and I. Nezbeda: Phys. Rev. E 79 (2009) 041141.

20) Y. J. Deng and H. W. J. Blote: Phys. Rev. E 72 (2005) 016126.

21) C. D. Lorenz, R. May and R. M. Ziff: J. Statist. Phys. 98 (2000) 961970 . 